\title{
COVID-19-associated severe immune thrombocytopenia
}

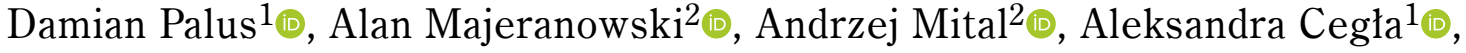

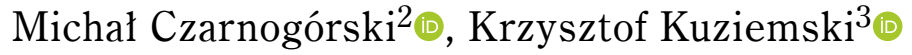 \\ ${ }^{1}$ The University Clinical Centre in Gdańsk, Gdańsk, Poland \\ ${ }^{2}$ Faculty of Medicine, Department of Haematology and Transplantology, Medical University of Gdańsk, \\ Gdańsk, Poland \\ ${ }^{3}$ Department of Pulmonology and Allergology, Faculty of Medicine, Medical University of Gdańsk, \\ Gdańsk, Poland
}

\begin{abstract}
Summary
The novel coronavirus disease (COVID-19) is not only a disease that affects the respiratory system but also a systemic disease resulting in various symptoms and complications. Haematological changes are common in patients with COVID-19 but reports of severe immune thrombocytopenia (IT) in patients with COVID-19 are rare. Hereby a case is presented of a 72-year-old male patient who developed severe, symptomatic IT a month after COVID-19. Thereinafter, aetiology, pathophysiology, diagnosis as well as treatment options were widely discussed.

\section{Key words: COVID-19, immune thrombocytopenia, thrombocytopenia, ITP, haematology, bleeding}

J. Transf. Med. 2021; 14: 131-133

\section{Introduction}

Immune thrombocytopenia (IT) can be primary - associated with antiplatelet autoantibodies or secondary - resulting from disturbed immune function due to neoplasia, pregnancy, chronic infections or autoimmune disorders (for example lupus erythematosus systemicus, antiphospholipid syndrome, acquired immunodeficiency syndrome). Primary thrombocytopenia (ITP) is characterized by reduced platelet production and/or increased platelet destruction resulting in a blood platelet count of less than $100 \times 10^{9} / \mathrm{L}$ and an increased risk of bleeding [1]. Clinical manifestations vary depending on the aetiology, the comorbidities and the severity of thrombocytopenia. It can range from an asymptomatic course to cutaneous purpura, epista- xis and even potentially fatal intracranial haemorrhage. The pathomechanism of ITP is postulated to be two-fold platelet destruction and inhibition of platelet production. The production of autoantibodies against platelet glycoproteins results in their opsonization and destruction, which leads to a decreased platelet count. The antibodies are directed against various platelet antigens, among which the most common are those directed against glycoprotein IIb/IIIa and Ib/IX [2]. Antibody-coated platelets are destroyed by macrophages in the reticuloendothelial system [3]. In addition to peripheral platelet destruction, inhibition of platelets production in bone marrow by antibodies directed against megakaryocytes also plays a role in ITP [3]. Severe acute respiratory syndrome coronavirus 2 (SARS-CoV-2) infection has been identified among

Adres do korespondencji: lek. Aleksandra Cegła, The University Clinical Centre in Gdańsk, Gdańsk, Poland, Dębinki Street 7 , 80-952 Gdańsk, e-mail: ola.cegla@gumed.edu.pl

Translation: lek. Alan Majeranowski

This article is available in open access under Creative Common Attribution-Non-Commercial-No Derivatives 4.0 International (CC BY-NC-ND 4.0) license, allowing to download articles and share them with others as long as they credit the authors and the publisher, but without permission to change them in any way or use them commercially. 
Table 1. Possible mechanisms leading to thrombocytopenia in COVID-19-induced IT [4]

Immune complex formation on platelet surface leading to clearance by the reticuloendothelial system

Expression of cryptic antigen on platelets leading to recognition by the immune system

Epitope spreading: no single anti-platelet antibody specificity to a particular glycoprotein

A direct effect of cytotoxic CD8+ T cells on platelets

Low or dysfunctional regulatory CD4+ T cells

Cross-presentation of exogenous antigens

PD-1 signalling

CRP binding to platelet phosphorylcholine residues, thereby facilitating lgG-mediated phagocytic responses against platelets

the various causes of IT [4]. The exact underlying mechanism in COVID-19-mediated IT is still being studied but among various possible explanations are molecular mimicry, cryptic antigen expression and epitope spreading. The different mechanisms have been presented in Table 1 [4]. Haematological abnormalities among COVID-19 patients are common and include a low lymphocyte count, prolonged activated partial thromboplastin time and elevated D-dimers levels. The prevalence of thrombocytopenia secondary to COVID-19 varies. Mild manifestations have been reported in up to one-third of the patients $[4,5]$. However, severe IT after COVID-19 is a rare clinical scenario and to the best of the authors' knowledge, only 45 similar cases have been described [4].

\section{Case description}

A 72-year-old male patient with a medical history of arterial hypertension and gout presented at the emergency department on 23.04.2021 with petechiae of the whole body, mucosal bleeding and mild epistaxis.

The patient first noticed purpuric rashes and mucosal bleeding two days earlier. He had been diagnosed with COVID19 at a different hospital on 27.03.2021 with mild symptoms - fever and cough. During that hospitalisation the platelet count was $224 \times 10^{9} / 1$. After a five-day stay with heparin and oxygen therapy, the patient was discharged home in a good state to conclude the 10-days isolation period.

Laboratory tests at the emergency department revealed isolated severe thrombocytopenia $1 \times$ $10^{9} / 1$ with no other additional deviations in blood morphology. Upon admission to the Haematology Department, a transfusion of one unit of gamma-irradiated leucodepleted platelet concentrate was ordered and a cycle of $4 \times 40 \mathrm{mg}$ dexamethasone was given. An extensive laboratory work-up was ordered. HIV, hepatitis B and C virus, CMV and parvovirus laboratory tests were all negative. The heparin-PF4 antibodies test was also negative. Platelet-induced IgM and IgA antibodies were elevated.

After the platelet transfusion, the platelet count went up to PLT $3 \times 10^{9} / 1$. The following day it was $21 \times 10^{2} / 1$ and the day after that $62 \times 10^{9} / 1$. On day 4 of hospitalization bone marrow biopsy was taken which did not reveal any myelodysplastic changes. The patient was discharged home on day 6 of hospitalization with a platelet count of $162 \times$ $10 \%$. During a follow-up visit 14 days later the platelet count was $156 \times 10^{9} / \mathrm{L}$.

\section{Discussion}

The occurrence of immune thrombocytopenia in patients with COVID-19 raises two fundamental questions. Firstly, is the SARS-CoV-2 infection an independent cause of IT or is it a triggering factor in patients with predispositions and specific comorbidities? Further studies are required to answer this question. A second important question is what is the most effective and safe treatment of IT in patients with COVID-19? Currently, available data shows that standard treatment protocols used in ITP are effective also in patients with COVID-19-associated IT [4]. In the present case, a cycle of $4 \times 40 \mathrm{mg}$ dexamethasone was an effective therapy. The patient developed IT after the SARS-CoV-2 infection was over, but in most of the described cases, IT occurred simultaneously with the COVID-19 infection. In that group of patients, treatment may be more challenging because the medication used in IT can negatively impact COVID-19 therapy. For example, high-dose dexamethasone pulses at the beginning of COVID-19 or in patients not receiving oxygen therapy can result in a worse prognosis [6]. Similar cases were treated successfully with intravenous immunoglobulins, 
glucocorticoids and thrombopoietin receptor agonists or different combinations [4] but the effectiveness of these treatment options needs to be evaluated in future randomised studies. While the underlying mechanisms of COVID-19-associated IT remain to be understood, the currently available data suggests that the diagnosis and treatment of COVID-19 induced IT should not differ from the updated recommendations for the treatment of ITP [7].

\section{References}

1. Rodeghiero F, Michel M, Gernsheimer T, et al. Standardization of terminology, definitions and outcome criteria in immune thrombocytopenic purpura of adults and children: report from an international working group. Blood. 2009; 113(11): 2386-2393, doi: 10.1182/blood-2008-07-162503, indexed in Pubmed: 19005182.
2. Kohli R, Chaturvedi S. Epidemiology and Clinical Manifestations of Immune Thrombocytopenia. Hamostaseologie. 2019; 39(3): 238-249, doi: 10.1055/s-0039-1683416, indexed in Pubmed: 30868551.

3. Arnold DM, Nazi I, Toltl LJ, et al. Antibody binding to megakaryocytes in vivo in patients with immune thrombocytopenia. Eur J Haematol. 2015; 95(6): 532-537, doi: 10.1111/ejh.12528, indexed in Pubmed: 25684257.

4. Bhattacharjee S, Banerjee M. Immune Thrombocytopenia Secondary to COVID-19: a Systematic Review. SN Compr Clin Med. 2020; 2(11): 2048-2058, doi: 10.1007/s42399-020-00521-8, indexed in Pubmed: 32984764.

5. Xu P, Zhou Qi, Xu J. Mechanism of thrombocytopenia in COVID-19 patients. Ann Hematol. 2020; 99(6): 1205-1208, doi: 10.1007/s00277-020-04019-0, indexed in Pubmed: 32296910.

6. Dexamethasone in Hospitalized Patients with Covid-19. New Engl J Med. 2021; 384(8): 693-704, doi: 10.1056/nejmoa2021436, indexed in Pubmed: 32678530.

7. Cunningham JM. Updated Recommendations for the Treatment of Immune Thrombocytopenia. Clin Adv Hematol Oncol. 2020; 18(8): 442-446, indexed in Pubmed: 32903242. 